\title{
Design of High Efficiency Illumination for LED Lighting
}

\author{
Yong-Nong Chang, ${ }^{1}$ Hung-Liang Cheng, ${ }^{2}$ and Chih-Ming Kuo ${ }^{1}$ \\ ${ }^{1}$ Department of Electrical Engineering, National Formosa University, Yunlin County 63201, Taiwan \\ ${ }^{2}$ Department of Electrical Engineering, I-Shou University, Kaohsiung 84001, Taiwan
}

Correspondence should be addressed to Yong-Nong Chang; ynchang@nfu.edu.tw

Received 11 September 2013; Accepted 6 October 2013

Academic Editor: Teen-Hang Meen

Copyright (C) 2013 Yong-Nong Chang et al. This is an open access article distributed under the Creative Commons Attribution License, which permits unrestricted use, distribution, and reproduction in any medium, provided the original work is properly cited.

\begin{abstract}
A high efficiency illumination for LED street lighting is proposed. For energy saving, this paper uses Class-E resonant inverter as main electric circuit to improve efficiency. In addition, single dimming control has the best efficiency, simplest control scheme and lowest circuit cost among other types of dimming techniques. Multiple serial-connected transformers used to drive the LED strings as they can provide galvanic isolation and have the advantage of good current distribution against device difference. Finally, a prototype circuit for driving $112 \mathrm{~W}$ LEDs in total was built and tested to verify the theoretical analysis.
\end{abstract}

\section{Introduction}

Green energy and high efficiency equipment have been developed rapidly to mitigate to global warming problem. In order to improve the efficiency of lighting equipments, light emitting diode (LED) has been paid attention and adopted as the light source. LED has the highest lighting efficiency among other lighting equipments. Furthermore, it possesses longer life cycle, faster dynamic response, smaller device volume and higher color rendering index. LED lighting equipments are widely used for automobiles [1-4], indoor lightings [5], outdoor lights [6,7], and street lamps recently. LED lighting equipments are look upon as the main lighting equipment in the future. In order to replace traditional lighting equipments, LED lighting equipments of high output power are developing rapidly in recent years. Performance of LED lighting equipment has been paid attention to, such as efficiency of LED drivers, device reliability, and dimming features. In this paper, multiple serial-connected transformers are used to overcome the problems mentioned above.

In order to drive the multiple serial-connected transformers, an inverter is required. Class-E serial resonant inverter will be used in this paper as main electric circuit. Class-E resonant inverter can achieve higher efficiency than ClassD resonant inverter $[8,9]$. In order to lower the usage of electricity for lightings during daytime, dimming features are seen as a way to save energy of lighting equipments. Conventional ways to achieve dimming feature is to use linear current-regulator to control LED lighting equipments individually [10]. Another way is to use PWM controller to achieve dimming control [11, 12], but PWM controller without current limitation function causes the shrink of life cycle of LED. Dimming switches of both ways do not have the ability of soft switching, which involves switching losses during dimming control. In this paper, integral-cycle control is used to achieve ZCS function of dimming switches, minimizing the power losses during dimming control.

There are several different control schemes to achieve same output power of an LED lighting equipment. This paper proposes three types of different dimming controls for multiple serial-connected transformers. Three types of dimming controls include synchronism dimming control, sequential dimming control, and single dimming control. The efficiency and control scheme are both compared in this paper to find out the best dimming control scheme.

\section{Topology and Analysis of Proposed Circuit}

To improve the efficiency of street lighting, Class-E resonant inverter is used in this paper. Class-E resonant inverter possesses higher efficiency than other types of inverter. 


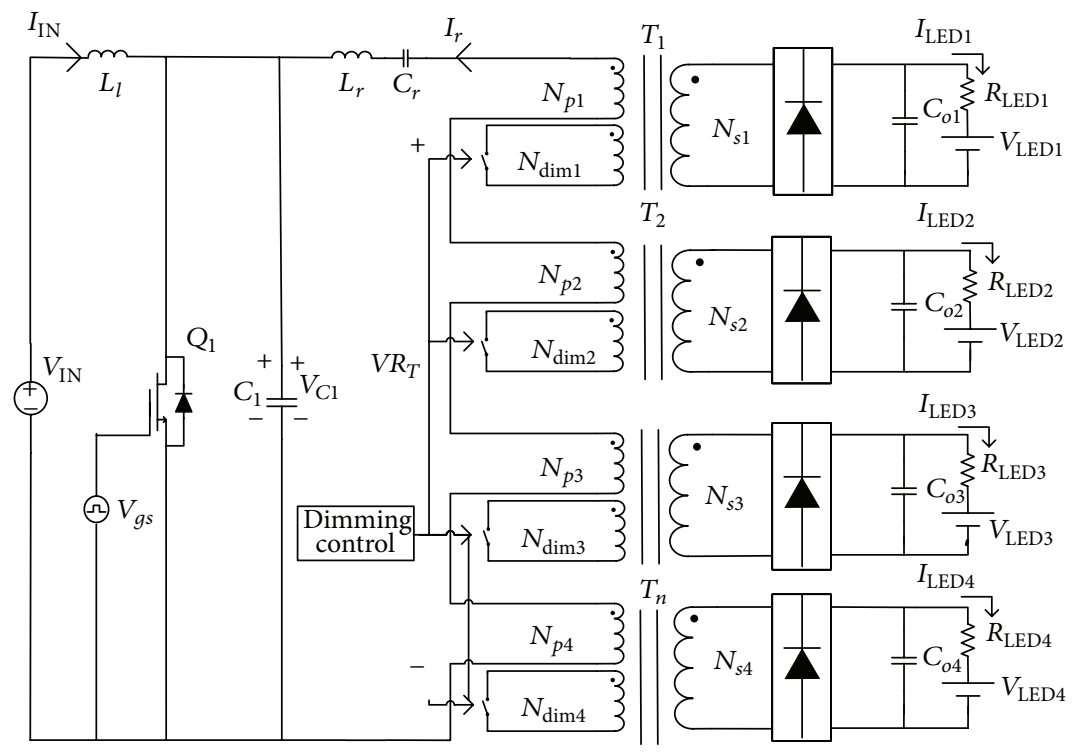

FIgURE 1: Proposed circuit in this paper.

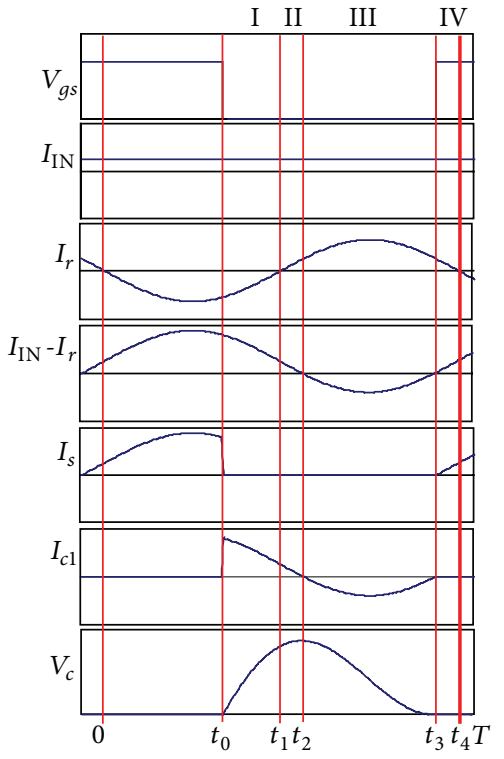

FIgURE 2: Theoretical optimum waveforms of Class-E resonant inverter.

The class-E resonant inverter in this paper will be operated in close to optimum mode to ensure ZVS function of the main switch at all time. Figure 1 shows the proposed circuit in this paper. Figure 2 shows the theoretical optimum waveforms of operating modes of class-E resonant inverter; it can be categorized into 4 modes as shown in Figure 3; the modes are described as follows.

2.1. Mode I: $t_{0}<t<t_{1}$. When switch S1 turns off, Mode I starts. As the switch of the resonant inverter turns off, current $I_{\mathrm{IN}}-I_{r}$ is in positive cycle. The current is flowing through the parallel capacitor $C_{1}$ and, voltage on $C_{1}$ is raised from zero.
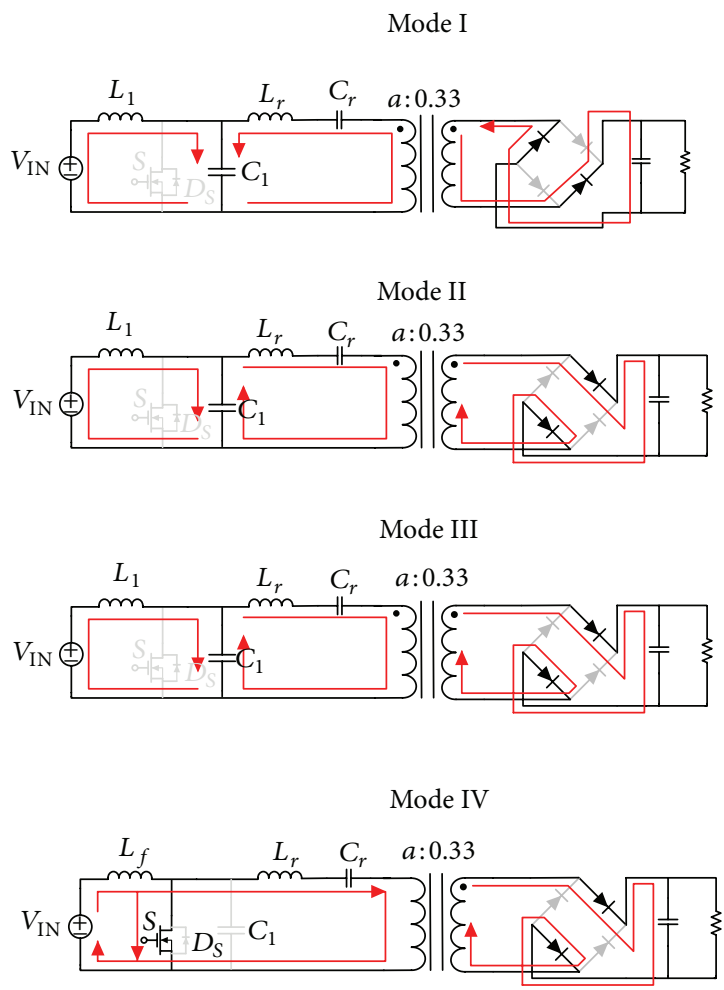

FIGURE 3: Operating modes of proposed circuit.

Mode I ends when the resonant current passes through zero and the circuit enters the next mode.

2.2. Mode II: $t_{1}<t<t_{2}$. Mode II starts when the resonant current enters positive cycle and ends when current of $C_{1}, I_{c 1}$ passes through zero point and then the circuit enters Mode III. 
2.3. Mode III: $t_{2}<t<t_{3}$. Mode III starts when the current $I_{\mathrm{IN}}-I_{r}$ passes through zero and enters negative cycle. The main switch of class-E resonant inverter remains off. The current $I_{\mathrm{IN}}-I_{r}$ enters negative cycle and so is the current of capacitor $C_{1}, I_{c 1}$. The voltage on the parallel capacitor $C_{1}$ begins to discharge. This mode ends when current $I_{\mathrm{IN}}-I_{r}$ passes through zero and enters the positive cycle.

2.4. Mode IV: $t_{3}<t<t_{4}$. Mode IV starts after current $I_{\mathrm{IN}}-I_{r}$ enters positive cycle. Parallel capacitor $C_{1}$ is completely discharged and the main switch turns on. Since the voltage applied on the parallel capacitor drops to zero and so is the voltage applied on the main switch. Therefore, the main switch of Class-E resonant inverter achieves zero-voltage switching (ZVS) function and reduces switching losses. This mode ends when the resonant current becomes negative and the circuit enters Mode I of the next high frequency cycle.

Reactance changes to resonant tank during dimming control might result in current variation. Current variation might result in malfunction of LED street lamp, which influences the safety of drivers, and it is not acceptable. Class-E resonant inverter must be well designed to prevent unacceptable current variation. Therefore, characteristics of proposed circuit are analyzed as follows.

To simplify the analysis, the circuit is simplified as in Figure 4. As shown in Figure 5, in order to simplify the parameters design, the loads are equivalent to the primary side of transformers $R_{p}$. The value of the input inductor will influence the ripple of input current which can be expressed as in (1). The value of DC input current can be estimated by (2). With a high load quality factor, almost all the harmonic contents and Dc components will be filtered by the resonant tank. Only the fundamental current at the switching frequency will be present in the load circuit. Therefore, the load resonant circuit can be analyzed using the fundamental component approximation. The fundamental voltage $V_{c 1}$ applied on parallel capacitor $C_{1}$ can be estimated by (3)

$$
\begin{gathered}
\Delta L_{1, p p}=\frac{V_{\mathrm{IN}}}{L_{1}} D T, \\
\Longrightarrow L_{1}=\frac{V_{\mathrm{IN}}}{\Delta i_{L, P P}} D T, \\
I_{\mathrm{IN}}=\frac{P}{V_{\mathrm{IN}}}, \\
V_{c 1} \approx \frac{2 \sqrt{2} \times V_{\mathrm{IN}}}{\pi} .
\end{gathered}
$$

During dimming control, the resonant current is impossible to be kept unchanged, and determination of the maximum loading current is essential. The variation of LED current is proportional to the variation of resonant current, and the resonant current is dominated by $Z_{\mathrm{LC}}$ and $R_{T}$. The minimum resonant current $I_{r \min }$ will occur at full load; minimum resonant current $I_{r \min }$ can be expressed as in (4), where $n$ is the number of LED lighting sets. When LED lighting sets are shorted during dimming control, the resonant current will rise to the maximum. The maximum resonant current $I_{r \max }$ occurs when only one equivalent resistance of LED set participates in the resonant tank. $I_{r \text { max }}$ can be expressed as in (5). With $T_{x}$ being defined as ratio of $Z_{\mathrm{LC}}$ and $R_{P}$ as expressed by (6), the resonant current ratio of $I_{r \text { min }}$ and $I_{r \text { max }}, K$ can be expressed as in (7)

$$
\begin{gathered}
I_{r \text { min }}=\frac{V_{c 1}}{\sqrt{\left(n R_{P}\right)^{2}+\left(Z_{\mathrm{LC}}\right)^{2}}}, \\
I_{r \max }=\frac{V_{c 1}}{\sqrt{\left(R_{P}\right)^{2}+\left(Z_{\mathrm{LC}}\right)^{2}}}, \\
Z_{\mathrm{LC}}=\left|T_{X} R_{P}\right|, \\
K \equiv \frac{I_{r \min }}{I_{r \max }}=\sqrt{\frac{\left(1+T_{X}^{2}\right)}{\left(n^{2}+T_{X}^{2}\right)}},
\end{gathered}
$$

where $K$ is the current ratio of $I_{r \text { min }}$ and $I_{r \max }$.

As shown in (8), according to Kirchhoff's voltage law (KVL), voltage of the resonant tank is equal to the sum of the voltage applied on resonant components and loads, and, wherein, the $R_{T}$ can be found by (9). Since the ratio of reactance $Z_{\mathrm{LC}}$ to $R_{p}$ has been found, voltage applied on single transformer $V_{R_{P}}$ with full load can be calculated by (10)

$$
\begin{gathered}
\left(V_{c 1}\right)^{2}=\left(V R_{T}\right)^{2}+\left(V_{Z_{\mathrm{LC}}}\right)^{2}, \\
R_{T}=n \times R_{P}, \\
V_{R_{P}}=\sqrt{\frac{V_{c 1}^{2}}{n^{2}+T_{x}^{2}} .}
\end{gathered}
$$

The specifications of LED lighting sets are given in Table 1. The operating voltage of LED lighting set at rate output, $V_{\text {lamp }}$, will be estimated by (11), where the $I_{\text {LED min }}$ can be estimated by (12)

$$
\begin{gathered}
V_{\text {lamp }}=V_{\mathrm{LED}}+I_{\mathrm{LED} \min } \cdot R_{\mathrm{LED}}, \\
I_{\mathrm{LED} \min }=I_{\mathrm{LED}} \times K .
\end{gathered}
$$

With the voltages of both sides of transformers being found out, turn ratio $N_{p} / N_{s}$ can be estimated by (13)

$$
a \equiv \frac{N_{P}}{N_{S}}=\frac{\pi \times V_{R_{P}}}{2 \sqrt{2} \times V_{\text {Lamp }}},
$$

where $a$ is the turn ratio in this paper.

Moreover, the resonant current during full load can be referred from the secondary side of transformer to the primary side; the RMS value of full load resonant current $I_{r \text { min }}$ can be estimated by (14)

$$
I_{r \min }=\frac{\pi \times I_{\mathrm{LED} \min }}{2 \sqrt{2} \times a} .
$$

Since all the components are ideal, there will be no power losses in transformers, and the input power is equal to the 


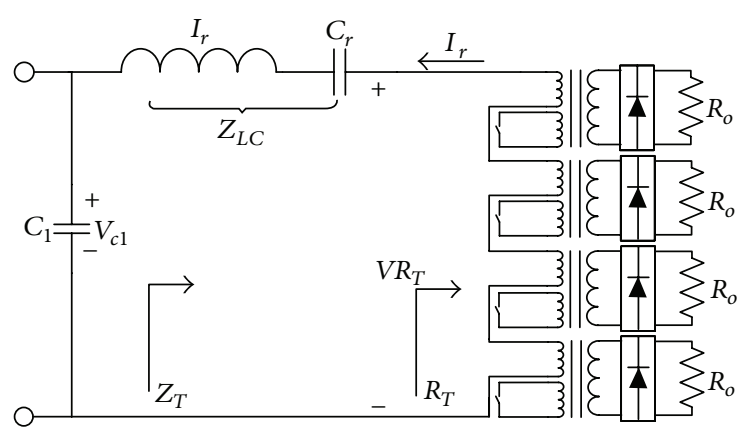

FIGURE 4: Simplified circuit.

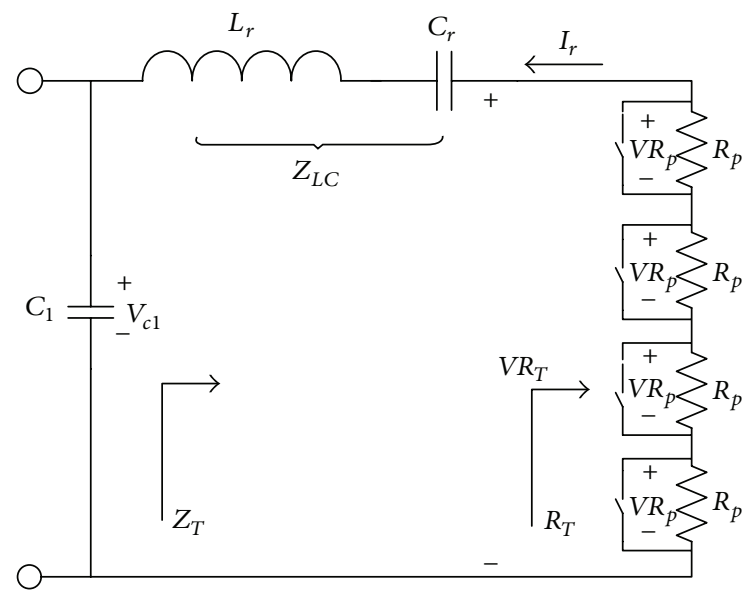

Figure 5: Equivalent power resistance $R_{p}$.

output power; therefore, the input power of primary side can be estimated by (15)

$$
P_{R_{P}}=I_{\text {LED min }} \times V_{\text {Lamp }} .
$$

With the input power $P_{R_{p}}$ and the resonant current of primary side $I_{r \text { min }}$, the equivalent power resistance can be estimated by (16)

$$
R_{P} \equiv \frac{P_{R_{P}}}{\left(I_{r \text { min }}\right)^{2}}=\frac{8 \times a^{2} \times V_{\text {Lamp }}}{\pi^{2} \times I_{\text {LED min }}},
$$

where $R_{p}$ is the equivalent power resistance in the primary side of transformers.

Since the value of $C_{1}$ influences whether the main switch of class-E resonant inverter has the capability of zero-voltage switching (ZVS) function or not, analysis of parallel capacitor $C_{1}$ is critical in this research. If the resonant current is defined as (17), the output power can be expressed as in (18). If RMS value of resonant current is expressed as in (19), the peak value of resonant current can be expressed as in (20). The current of parallel capacitor $C_{1}, I_{c 1}$, is expressed as in (21). The instantaneous voltage of parallel capacitor $C_{1}, V_{c 1}(t)$, is expressed as in (22). $V_{c 1}\left(t_{3}\right)$ will drop to zero at time instant $t_{3}$ as expressed by (23). Besides, $V_{c 1}\left(t_{3}\right)$ can be expressed as in (24). Therefore, the angle, $\Phi$, by which the resonant voltage leads the resonant current can be found by deducing (24). The mean voltage of $C_{1}, V_{c 1 a v}$ will be equal to the input voltage $V_{\text {IN }}$ as in (25). The mean voltage of $C_{1}, V_{c 1 a v}$, can be expressed as in (26). Therefore, the value of $C_{1}$ can be rewritten as in (27), and the value can be found by deriving (27)

$$
\begin{gathered}
I_{r}=I_{o} \sin (\omega t-\Phi), \\
P=I_{r}{ }^{2} \times R_{T},
\end{gathered}
$$

$$
\begin{gathered}
I_{r(\mathrm{rms})}=\sqrt{\frac{4 \times I_{\mathrm{LED} \text { min }} \times V_{\mathrm{Lamp}}}{R_{T}},} \\
I_{o(\text { peak })}=\sqrt{\frac{8 \times I_{\mathrm{LED} \text { min }} \times V_{\mathrm{Lamp}}}{R_{T}}}, \\
i_{c 1}(t)=I_{\mathrm{IN}}-I_{o} \sin (\omega t-\Phi), \\
V_{c 1}(t)=\frac{1}{C_{1}} \int_{t_{0}}^{t}\left[I_{\mathrm{IN}}-I_{o} \sin (\omega t-\Phi)\right] d t \\
=\frac{1}{C_{1}}\left[I_{\mathrm{IN}} \times t+\frac{I_{o}}{\omega} \cos (\omega t-\Phi)-\frac{I_{o}}{\omega} \cos \Phi\right], \\
V_{c 1}\left(t_{3}\right)=0,
\end{gathered}
$$$$
V_{c 1}\left(t_{3}\right)=\frac{1}{C_{1}} \int_{t_{o}}^{t_{3}}\left[I_{D C}-I_{o} \sin (\omega t-\Phi)\right] d t
$$$$
=\frac{1}{C_{1}}\left[(1-D) T \times I_{\mathrm{IN}}+\frac{I_{o}}{\omega} \cos (2 D \pi+\Phi)\right.
$$$$
\left.-\frac{I_{o}}{\omega} \cos \Phi\right]
$$$$
V_{c 1 a v}=V_{\mathrm{IN}}
$$

$$
\begin{aligned}
& V_{c 1 a v}=\frac{1}{T} \int_{t_{0}}^{(1-D) T} V_{c 1}(t) d t \\
& =\frac{1}{C_{1}}\left\{\frac{(1-D)^{2} T}{2} I_{\mathrm{IN}}-\frac{(1-D) T}{2 \pi} \cos \Phi \times I_{o}\right. \\
& \left.+\frac{T}{4 \pi^{2}} I_{o}[\sin \Phi-\sin (2 D \pi+\Phi)]\right\}, \\
& C_{1}=\frac{1}{V_{\mathrm{IN}}}\left\{\frac{(1-D)^{2} T}{2} I_{\mathrm{IN}}-\frac{(1-D) T}{2 \pi} \cos \Phi \times I_{o}\right. \\
& \left.+\frac{T}{4 \pi^{2}} I_{o}[\sin \Phi-\sin (2 D \pi+\Phi)]\right\} \text {. }
\end{aligned}
$$

Using the parameters of resonant frequency of resonant tank $f_{r}$ and switching frequency $f_{s}$ defined in Table 1 , the reactance $Z_{\mathrm{LC}}$ defined as in (28) and resonant components $C_{r}$ 
TABLE 1: Specifications of proposed circuit.

\begin{tabular}{lc}
\hline Input voltage, $V_{\mathrm{IN}}$ & $140 \mathrm{~V}$ \\
Current ratio, $K$ & 0.7 \\
Turn ratio, $a$ & 0.30 \\
Turn ratio of dimming circuit & 0.125 \\
Switching frequency, $f_{s}$ & $50 \mathrm{kHz}$ \\
Resonant frequency, $f_{r}$ & $40 \mathrm{kHz}$ \\
Maximum loading current of LED, $I_{\mathrm{LED}}$ & $350 \mathrm{~mA}$ \\
Operating voltage of LED, $V_{\mathrm{LED}}$ & $78.8 \mathrm{~V}$ \\
Total output power, $P_{o}$ & $112 \mathrm{~W}$ \\
Value of output capacitor, $C_{\text {on }}$ & $330 \mu \mathrm{F}$ \\
\hline
\end{tabular}

TABle 2: Parameters deduced from design procedures.

\begin{tabular}{lc}
\hline Input inductor, $L_{1}$ & $10 \mathrm{mH}$ \\
Voltage apply on $C_{1}, V_{c 1}$ & $126 \mathrm{~V}$ \\
Magnification of resistance, $T_{X}$ & 3.66 \\
Equivalent power resistance, $R_{T}$ & $118 \Omega$ \\
Voltage apply on a single transformer, $V_{R_{P}}$ & $23.24 \mathrm{~V}$ \\
Minimum resonant current, $I_{r \text { min }}$ & $0.9 \mathrm{~A}$ \\
Minimum loading current of LEDs, $I_{\mathrm{LED} \mathrm{min}}$ & $280 \mathrm{~mA}$ \\
Value of parallel capacitor, $C_{1}$ & $20.5 \mathrm{nF}$ \\
Value of resonant capacitor, $C_{r}$ & $49 \mathrm{nF}$ \\
Value of resonant inductor, $L_{r}$ & $321 \mu \mathrm{H}$ \\
\hline
\end{tabular}

and $L_{r}$ can be solved by deducing simultaneous (28), where $K$ is the resonant current ratio of $I_{r \text { min }}$ and $I_{r \text { max }}$

$$
\begin{aligned}
& Z_{\mathrm{LC}}=\omega_{s} L_{r}-\frac{1}{\omega_{s} C_{r}}, \\
& \omega_{r}=2 \pi f_{r}=\frac{1}{\sqrt{L_{r} C_{r}}} .
\end{aligned}
$$

\section{Experimental Results}

In this paper, a high efficiency street lighting is proposed. Table 1 shows the specification of the proposed circuit, following the design procedures in the previous section. Parameters can be deduced as in Table 2. Figure 6 shows the waveforms of the Class-E resonant inverter during full load using the parameters listed in Table 2. Experimental waveforms show that the experimental results are in good consistency with the theoretical prediction.

Figures 7 and 8 show the waveforms which indicate that the main switch is able to achieve ZVS function in both full load and single load. Figure 7 shows the voltage waveforms of $V_{g s}$ and $V_{c 1}$ during single load and Figure 8 shows the voltage waveforms of $V_{g s}$ and $V_{c 1}$ during full load. Resonant current will flow through the body diode of the main switch before it turns on. It can be clearly seen that the main switch of the Class-E resonant inverter has achieved ZVS function in both full load and single load as demanded.

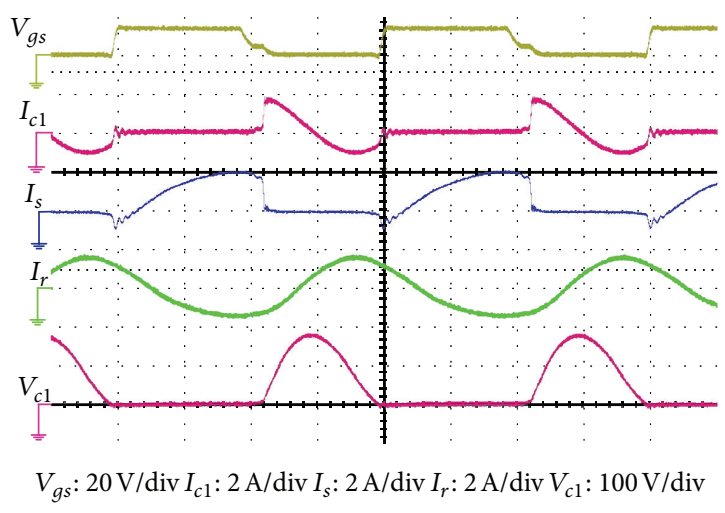

FIGURE 6: Experimental waveforms of Class-E resonant inverter.

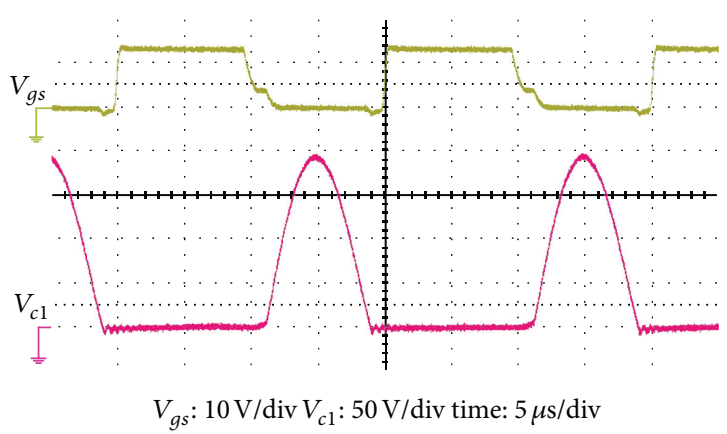

Figure 7: Waveforms of ZVS function of the main switch of Class-E resonant inverter during single load.

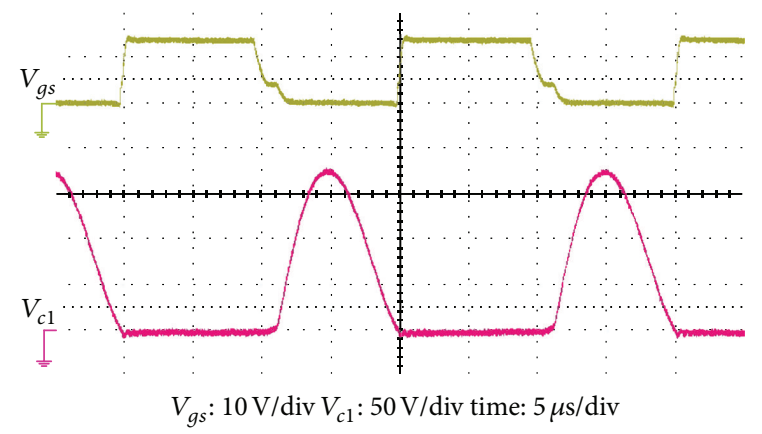

FIGURE 8: Waveforms of ZVS function of the main switch of Class-E resonant inverter during full load.

\section{Discussions of Different Types of Dimming Control}

With integral-cycle control being used in this paper to implement the dimmer design, wide range and precise dimming control can be achieved. During dimming control, reactance changes will occur. There are several ways to achieve same output effect with different dimming control techniques. The relationship between different dimming techniques and reactance changes will be discussed in this section. The frequency of dimming signal $V_{g s \text {-dim }}$ is $500 \mathrm{~Hz}$ and switching frequency of the Class-E resonant inverter is $50 \mathrm{kHz}$. Dimming range is made possible from $0.25 \%$ to $100 \%$. Since street lamps are 


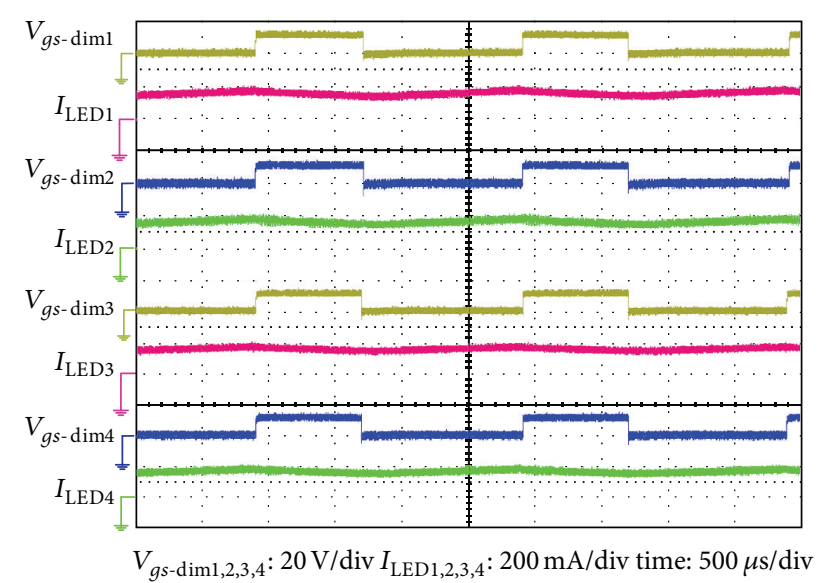

FIGURE 9: $60 \%$ output of synchronism dimming control.

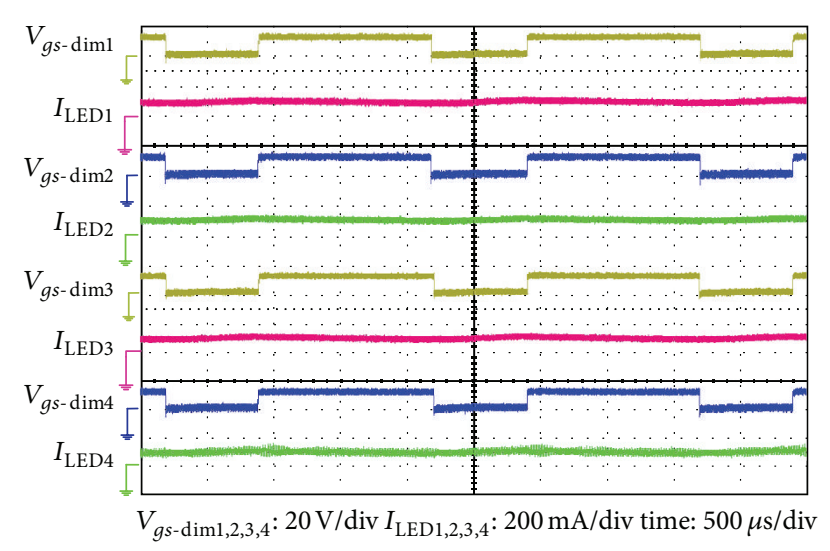

FIGURE 10: 35\% output of synchronism dimming control.

operated at rate output, generally, they do not require wide range of dimming control. The dimming control of 35\% to $100 \%$ rated power is proposed in this paper. Output capacitors with great value are used in this paper to limit the effect of current variation occurrence during dimming control.

4.1. Synchronism Dimming Control. The first way to control the LED lighting set is to control all the lighting sets simultaneously, while the LED lighting sets are able to produce the same amount of output power. But in this way, the reactance will change at any time, leading to a huge reactance change to the whole system during dimming control.

Figure 9 shows $60 \%$ rated power; Figure 10 shows 35\% rated power. Theoretically, the mean value of loading current $I_{\mathrm{LED} n}$ under $85 \%$ output of dimming control is about $238 \mathrm{~mA}$, while it is $168 \mathrm{~mA}$ under $60 \%$ output of dimming control, $98 \mathrm{~mA}$ under $35 \%$ output of dimming control, and $56 \mathrm{~mA}$ under $20 \%$ output of dimming control.

4.2. Sequential Dimming Control. To avoid reactance changes at any time and leading huge reactance to whole system, sequential dimming control is proposed. LED lighting sets are separated into 2 groups, with $1 \mathrm{~ms}$ delay of each group.

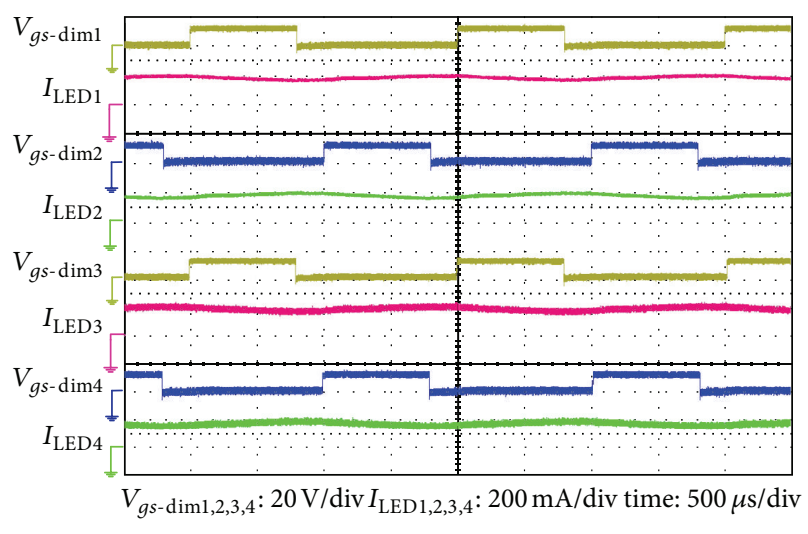

FIGURE 11: $60 \%$ output of sequential dimming control.

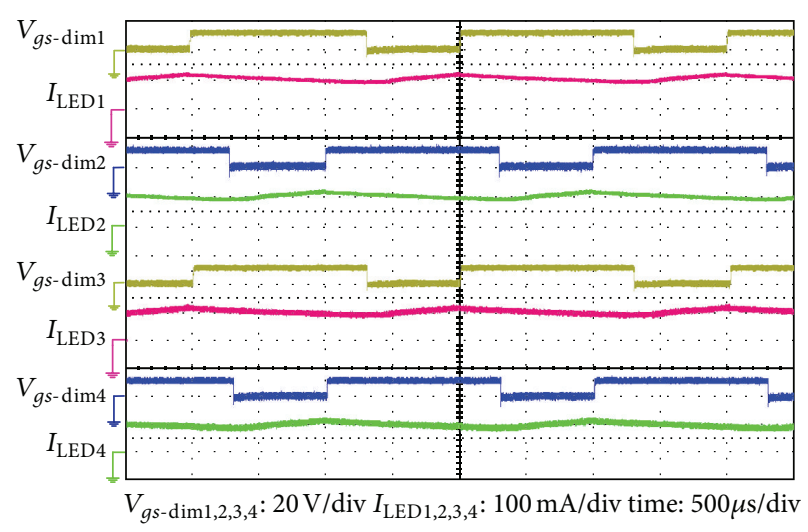

FIGURE 12: 35\% output of sequential dimming control.

The reactance changes of each LED lamp can be equally shared in different periods, which lower the reactance changes comparing to synchronism dimming control. Figure 11 shows $60 \%$ output of dimming control; Figure 12 shows $35 \%$ output of dimming control. In these ways, four sets of integral control circuits must be required, and control scheme is complicated as well.

4.3. Single Dimming Control. Last technique is single dimming control. Only one LED lighting set is in charge of detail dimming control while the rest of the LED lighting sets are kept either on or off. In this way, only one set needs the Integral-Cycle Control circuit, which can reduce the circuit cost and simplify the control scheme, the reactance change is the lowest as well.

Figure 13 shows 60\% output of dimming control; Figure 14 shows $35 \%$ output of dimming control.

Finally, we compare the efficiency of the three types of dimming controls, Figure 15 shows the efficiency of the three types of dimming controls. Single dimming control has the best efficiency among the three types; it requires one integralcycle control circuit only, reduces reactance changes during dimming control, and is a comparatively simplified dimming control scheme. Figure 16 shows the lumen curve of single dimming control from $35 \%$ to $100 \%$ output. 


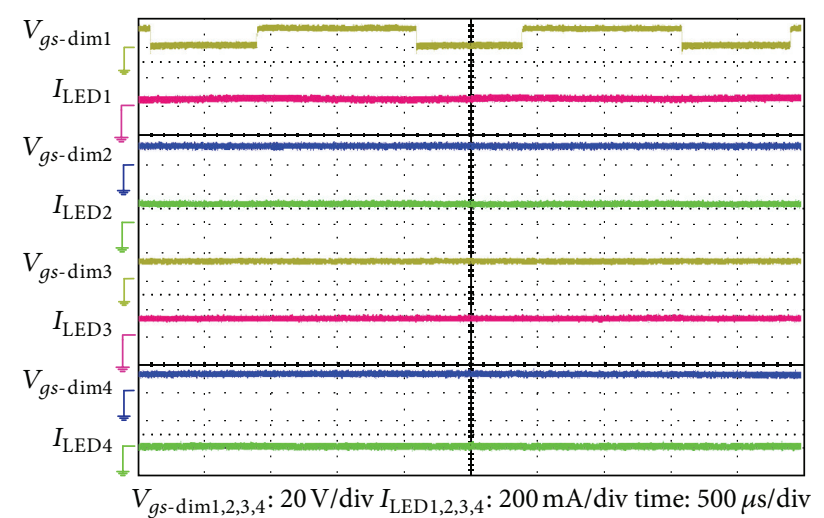

FIGURE 13: $60 \%$ output of single dimming control.

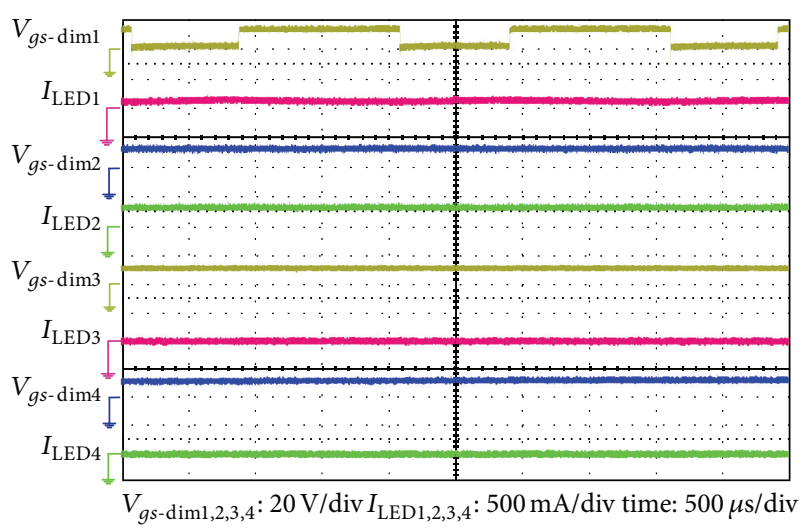

FIGURE 14: 35\% output of single dimming control.

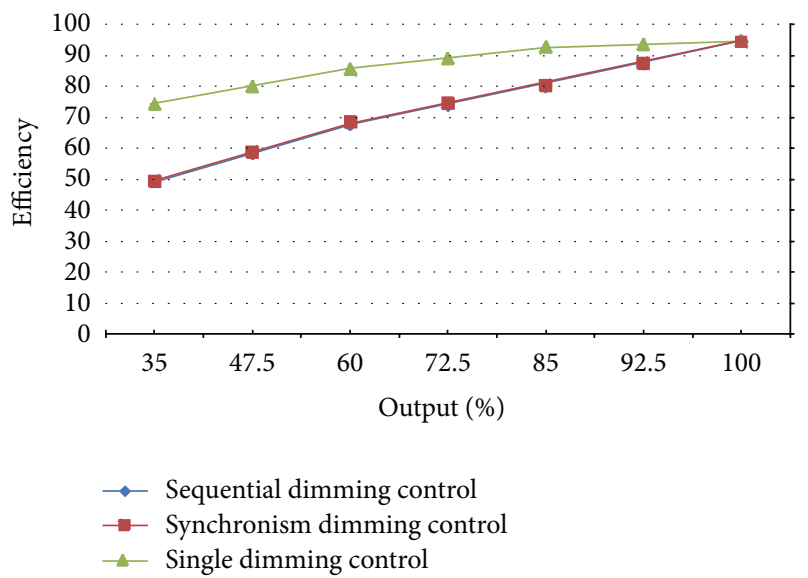

FIGURE 15: Efficiency of three types of dimming controls.

\section{Conclusion}

Via precise design of the Class-E resonant inverter, LED street lamps can prevent damage from current variation during dimming control. From experimental results, multiple serial-connected transformers have successfully solved the problems of power isolation and current uniformity. Single dimming control is the best way of achieving dimming

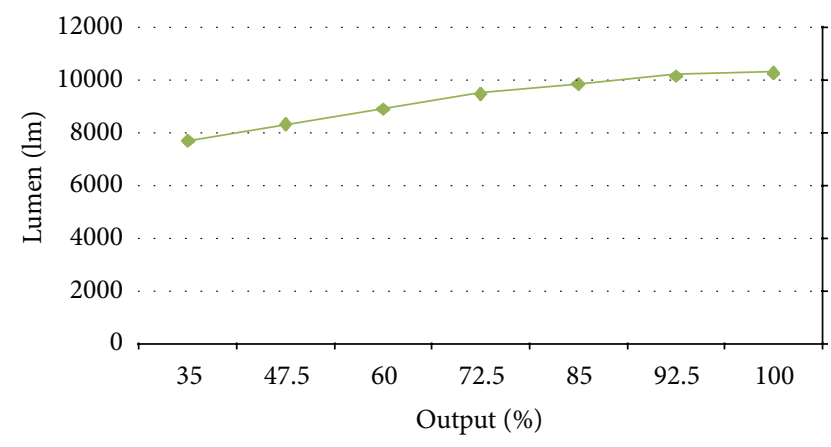

FIGURE 16: Lumen curve of single dimming control.

feature. Not only did it reduce the reactance changes during dimming control, but it also achieves comparatively the highest efficiency as well, leading to curtailing circuit cost and simplifying the control scheme. Finally, an $112 \mathrm{~W}$ high efficiency illumination for LED street lighting is developed, and efficiency can maintain $94 \%$ and $10270 \mathrm{~lm}$ at rate output.

\section{Conflict of Interests}

The authors declare that there is no conflict of interests regarding the publication of this paper.

\section{Acknowledgment}

This research is supported by the National Science Council, R.O.C., under Contract NSC 101-2221-E-150 -067-MY2.

\section{References}

[1] S. Saponara, G. Pasetti, N. Costantino, F. Tinfena, P. D’Abramo, and L. Fanucci, "A flexible LED driver for automotive lighting applications: IC design and experimental characterization," IEEE Transactions on Power Electronics, vol. 27, no. 3, pp. 10711075, 2012.

[2] S. Jang and M. W. Shin, "Thermal analysis of LED arrays for automotive headlamp with a novel cooling system," IEEE Transactions on Device and Materials Reliability, vol. 8, no. 3, pp. 561-564, 2008.

[3] D. Gacio, J. Cardesin, E. L. Corominas, J. M. Alonso, M. DallaCosta, and A. J. Calleja, "Comparison among power LEDs for automotive lighting applications," in Proceedings of the IEEE Industry Applications Society Annual Meeting, (IAS '08), vol. 93, pp. 1204-1223, Alberta, Canada, October 2008.

[4] I. Takai, S. Ito, K. Yasutomi, K. Kagawa, M. Andoh, and S. Kawahito, "LED and CMOS image sensor based optical wireless communication system for automotive applications," IEEE Photonics Journal, vol. 5, Article ID 6801418, 2013.

[5] S. Y. Jung, S. Hann, and C. S. Park, "TDOA-based optical wireless indoor localization using LED ceiling lamps," IEEE Transactions on Consumer Electronics, vol. 57, no. 4, pp. 15921597, 2011.

[6] S. Moon, G. B. Koo, and G. W. Moon, "A new control method of interleaved single-stage flyback AC-DC converter for outdoor LED lighting systems," IEEE Transactions on Power Electronics, vol. 28, pp. 4051-4062, 2013. 
[7] M. Wada, T. Yendo, T. Fujii, and M. Tanimoto, "Performance enhancement of outdoor visible-light communication system using selective combining receiver," IET Optoelectronics, vol. 3, no. 1, pp. 30-39, 2009.

[8] T. Suetsugu and M. K. Kazimierczuk, "Design procedure of Class-E amplifier for off-nominal operation at $50 \%$ duty ratio," IEEE Transactions on Circuits and Systems, vol. 53, no. 7, pp. 1468-1476, 2006.

[9] I. Boonyaroonate and S. Mori, "Analysis and design of class E isolated $\mathrm{dc} / \mathrm{dc}$ converter using class E low dv/dt PWM synchronous rectifier," IEEE Transactions on Power Electronics, vol. 16, no. 4, pp. 514-521, 2001.

[10] H. J. Chiu, H. M. Huang, H. T. Yang, and S. J. Cheng, "An improved single-stage flyback PFC converter for highluminance lighting LED lamps," International Journal of Circuit Theory and Applications, vol. 36, no. 2, pp. 205-210, 2008.

[11] H. J. Chiu and S. J. Cheng, "LED backlight driving system for large-scale LCD panels," IEEE Transactions on Industrial Electronics, vol. 54, no. 5, pp. 2751-2760, 2007.

[12] Y. T. Huang, H. C. Hsiao, Y. H. Liu, C. R. Lee, and L. L. Lee, "A novel constant-power control for metal-halide lamp electronic ballasts with dimming capability," IEEE Transactions on Plasma Science, vol. 38, no. 6, pp. 1482-1488, 2010. 

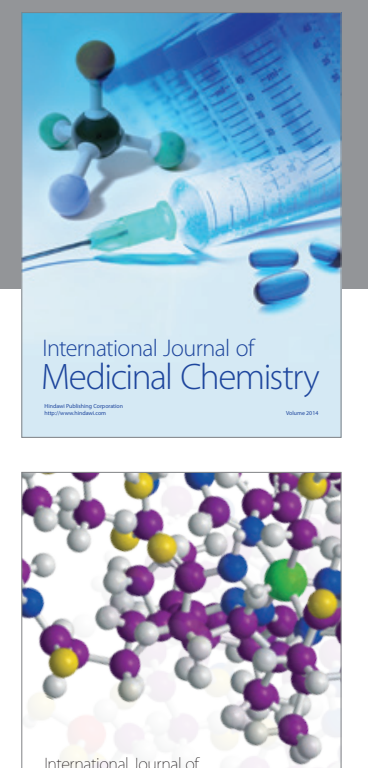

\section{Carbohydrate} Chemistry

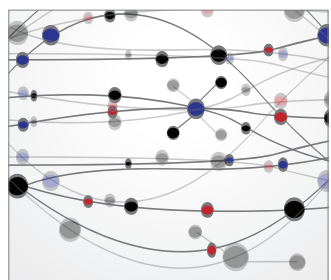

The Scientific World Journal
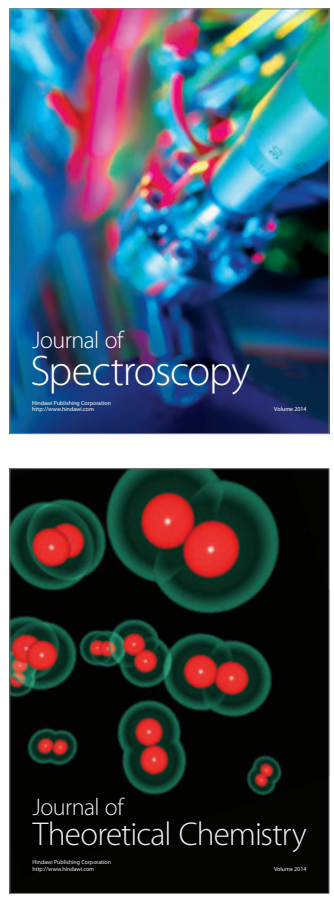
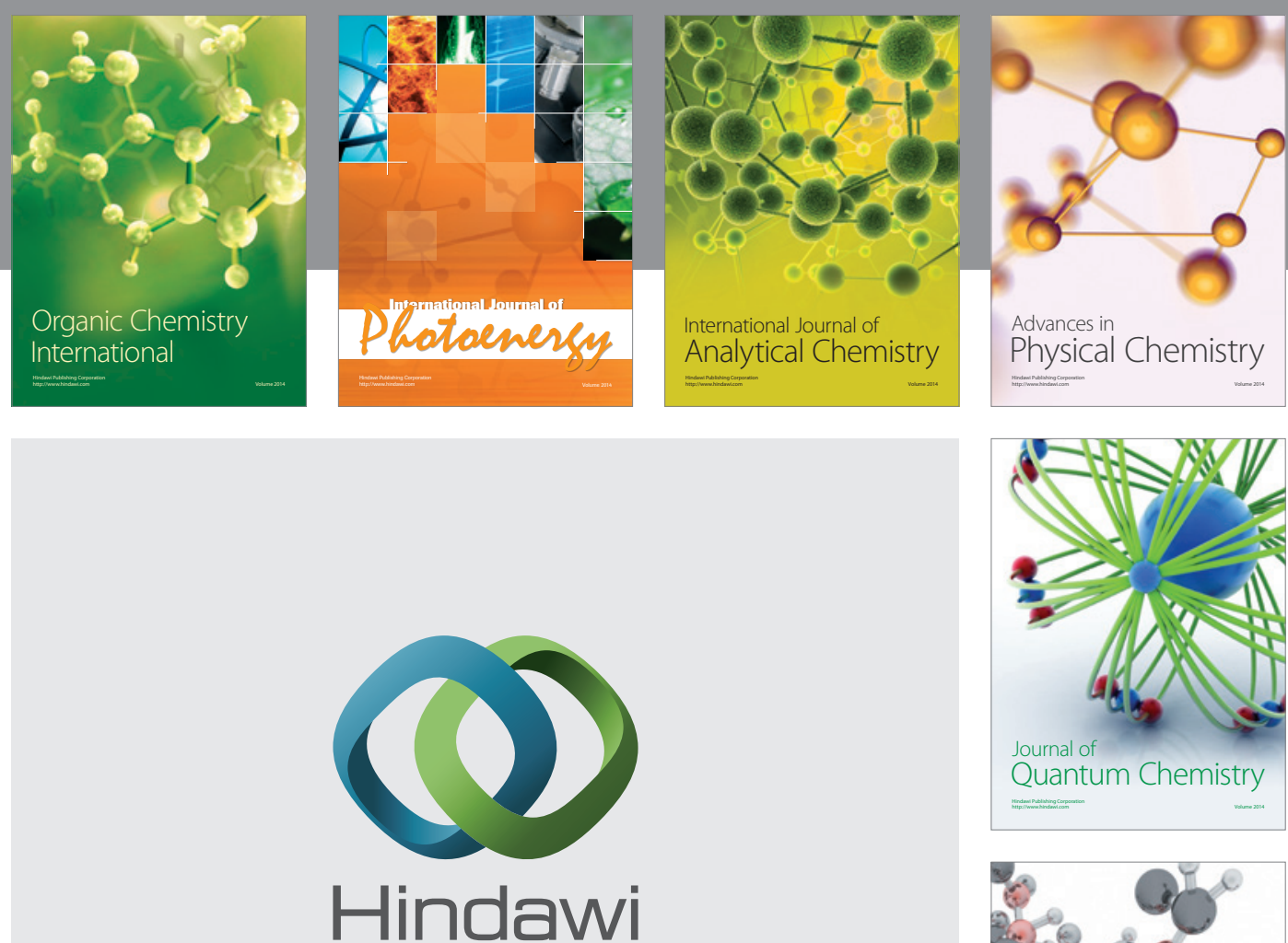

Submit your manuscripts at

http://www.hindawi.com

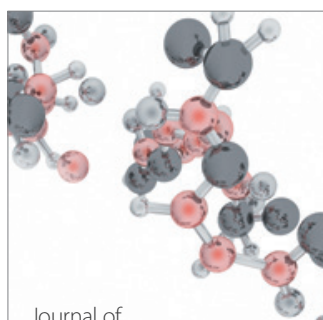

Analytical Methods

in Chemistry

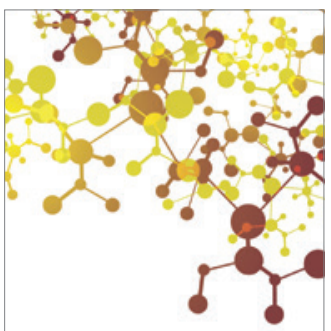

Journal of

Applied Chemistry

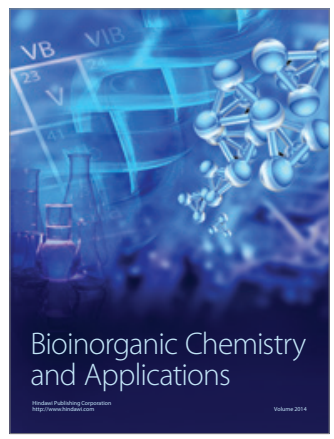

Inorganic Chemistry
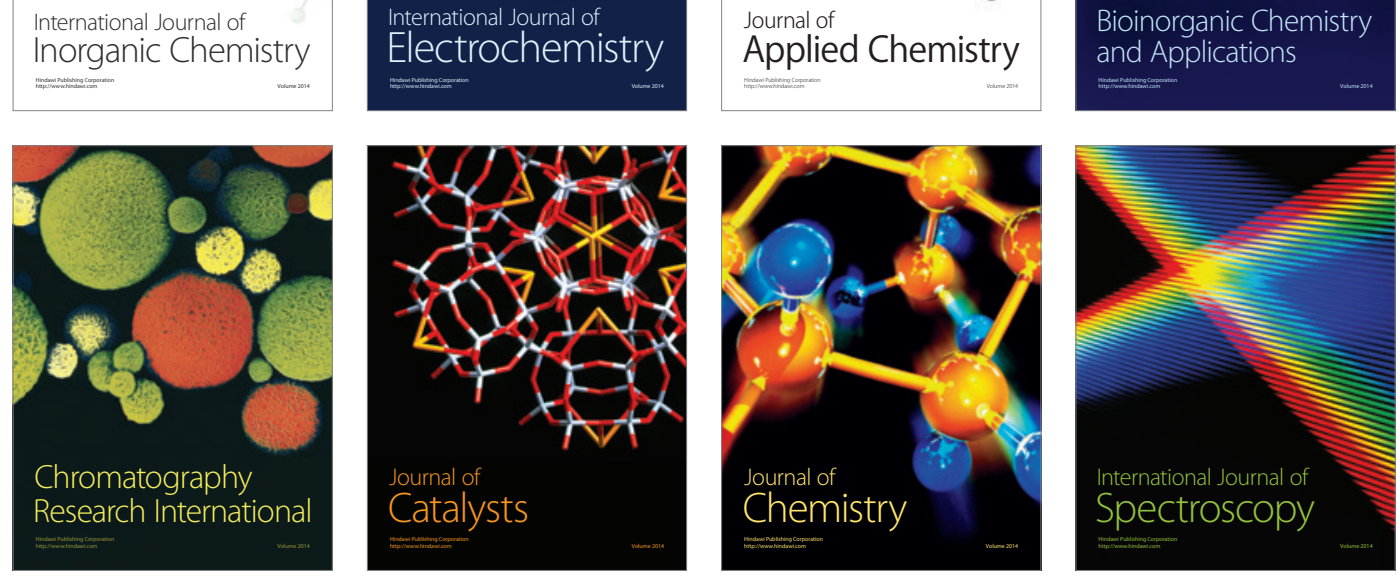